\title{
Fat-rich diet-induced obese mice show premature immunosenescence
}

\author{
O. Hernández ${ }^{1}$, L. E. Díaz ${ }^{2}$, G. Baccan 2,3 , A. Gheorge ${ }^{2}$, B. Zapatera ${ }^{2}$, A. Marcos ${ }^{2}$ and \\ M. De La Fuente \\ ${ }^{1}$ Department of Physiology. Faculty of Biology, Complutense University of Madrid, ${ }^{2}$ Department of Metabolism and \\ Nutrition. Institute of Food Science, Technology and Nutrition (ICTAN), Spanish National Research Council (CSIC), \\ Madrid, Spain and ${ }^{3}$ Institute of Health Sciences (Federal University of Bahia), Salvador, Brazil
}

Obesity, a modern pandemic, is associated with a chronic grade of oxidation-inflammation, which may produce an acceleration of the immune system impairment. Since the age-related changes on the immune system seem to be similar to those found in obese subjects, we have proposed obesity as a model of premature immunosenescence ${ }^{(1)}$. This fact could be one of the reasons to develop an early ageing rate related to obesity as well as a high risk of morbidity and mortality. The aim of the present study was to confirm the premature immunosenescence of obese adult mice fed with a fat-rich diet, analysing several functions in peritoneal immune cells such as chemotaxis, NK activity and lymphocyte proliferation, which have been proposed as biomarkers of the ageing rate and predictors of longevity ${ }^{(2)}$. Young female ICR mice $(n=16)$ (14 weeks old) were divided into two groups: 1$)$ Controls (C): fed ad libitum with a maintenance diet (Harlan) and 2) fed ad libitum with a high fat diet (HFD) containing $60 \%$ fat (Harlan). After 14 weeks (when animals were adults and HFD group developed obesity features), mice were sacrificed and peritoneal cell suspensions were obtained from both groups (HFD and C). The following functions were evaluated in peritoneal leukocytes: lymphocyte and macrophage chemotaxis, phagocytosis of inert particles by macrophages, anti-tumour NK activity and lymphocyte proliferative response to mitogens such as ConA and LPS (Table). The results showed that chemotaxis capacity of both macrophages and lymphocytes, NK activity and the proliferative response of lymphocytes to both mitogens (ConA and LPS) were significantly lower in HFD mice than in C group. Since an age-related decrease of these functions has been already shown ${ }^{(2)}$ ) we can confirm that adult mice developing obesity after having been fed with a fat-rich diet during their adolescence show a premature immunosenescence, which predispose them in the adulthood to a high risk of infection and tumours. This premature impairment of key immune functions could be related to a shorter life span, suggesting a status of premature ageing in obese mice.

\begin{tabular}{|c|c|c|c|c|}
\hline \multirow[b]{2}{*}{ FUNCTIONS } & \multirow[b]{2}{*}{$\mathrm{N}$} & \multirow{2}{*}{$\frac{\mathrm{CONTROL}}{\text { Mean } \pm \mathrm{SD}}$} & \multirow{2}{*}{$\frac{\mathrm{HFD}}{\text { Mean } \pm \mathrm{SD}}$} & \multirow[b]{2}{*}{$P$} \\
\hline & & & & \\
\hline Macrophage chemotaxis Index & 8 & $963 \pm 113$ & $554 \pm 64$ & 0.001 \\
\hline Lymphocyte chemotaxis Index & 8 & $1005 \pm 109$ & $620 \pm 100$ & 0.001 \\
\hline Phagocytosis Index & 8 & $251 \pm 69$ & $231 \pm 49$ & 0.507 \\
\hline Phagocytic efficacy Index & 8 & $68 \pm 6$ & $64 \pm 10$ & 0.329 \\
\hline Natural killer activity (\% Lysis) & 8 & $67 \pm 23$ & $46 \pm 12$ & 0.010 \\
\hline Proliferative response ConA & 8 & $6551 \pm 1299$ & $2773 \pm 1035$ & 0.001 \\
\hline cpm) $L P S$ & 8 & $8521 \pm 2394$ & $2394 \pm 1736$ & 0.001 \\
\hline
\end{tabular}

This work was supported by MICINN (BFU2011-30336), UCM (910379ENEROINN) grants, RETICEF (RD06/0013/0003) from ISCIII and BTSA (The PRONAOS Study, CDTI 2008 1114).

1. De la Fuente M \& De Castro NM (2012) Curr Immunol Rev 8, 63-75.

2. De la Fuente M \& Miquel J (2009) Current Pharm Des. 15 (26), 3003-3026. 